\title{
The novel pathogenic variant in the LMNA gene in a four-generation family with sudden deaths and cardiomyopathy: Utility of molecular autopsy
}

\author{
Agnieszka Zienciuk-Krajka' ${ }^{1}$ Magdalena Chmara²-4, Monika Lica-Gorzyńska , Karolina Dorniak \\ Joanna Kwiatkowska7 , Jacek Kowalski ${ }^{8}$, Damian Kaufmann' ${ }^{7}$, Ludmiła Daniłowicz-Szymanowicz', \\ Grzegorz Raczak'
}

\author{
'Department of Cardiology and Electrotherapy, Medical University of Gdansk, Gdańsk, Poland \\ 2Department of Biology and Genetics, Medical University of Gdansk, Gdańsk, Poland \\ ${ }^{3}$ Laboratory of Clinical Genetics, University Clinical Center, Gdańsk, Poland \\ ${ }^{4}$ Translational Medicine Center, Medical University of Gdansk, Gdańsk, Poland \\ ${ }^{5}$ Cardiology Unit, JK Łukowicz Specialist Hospital, Chojnice, Poland \\ ${ }^{6}$ Department of Noninvasive Cardiac Diagnostics, Medical University of Gdansk, Gdańsk, Poland \\ 'Department of Pediatric Cardiology and Congenital Heart Defect, Medical University of Gdansk, Gdańsk, Poland \\ ${ }^{8}$ Department of Pathomorphology, Medical University of Gdansk, Gdańsk, Poland
}

\author{
Correspondence to: \\ Agnieszka Zienciuk-Krajka, \\ $\mathrm{MD}, \mathrm{PhD}$, \\ Department of Cardiology and \\ Electrotherapy, \\ Medical University of Gdansk \\ Dębinki 7, 80-952 Gdańsk, \\ Poland, \\ phone: +48 585844760 , \\ e-mail: agzien@gumed.edu.pl \\ Copyright by the Author(s), 2021 \\ Kardiol Pol. 2021; \\ 79 (12): 1368-1371; \\ DOI: 10.33963/KP.a2021.0148 \\ Received: \\ August 12, 2021 \\ Revision accepted: \\ October 27, 2021 \\ Published online: \\ October 30, 2021
}

\section{INTRODUCTION}

Cardiac laminopathies involve electromechanical alterations of cardiomyocytes caused by genetic variants in the LMNA gene [1]. Cardiac presentations include dilated cardiomyopathy (DCM), atrioventricular block (AVB), atrial and ventricular tachyarrhythmias (VTA), and sudden cardiac death (SCD) [1]. Besides overt DCM and heart failure (HF), cardiac laminopathy may also present as systolic dysfunction without left ventricular (LV) dilatation (HNDC, hypokinetic non-dilated cardiomyopathy) or result in myocardial scarring detectable on cardiac magnetic resonance (CMR) [1, 2]. Recognition of cardiolaminopathy has an ominous prognosis due to the propensity for malignant VTA that precedes ventricular dilation and dysfunction [3]. Several tools for SCD risk prediction were proposed, each incorporating results of genetic testing [3-6]. However, clinical use of molecular data is frequently limited by a lack of credible information on the pathogenicity of genetic variants, previously deemed pathogenic based on reports of single cases [7].

This study presents a novel pathogenic variant in the LMNA gene in a large 4-generation Polish family.

\section{METHODS}

The study was approved by the local Ethics Committee, and patients gave written informed consent for participation in this study, genetic testing, and the use of their clinical data.

The pedigree tree of 37 individuals from a 4-generation Polish family with a history of $\mathrm{SCD}, \mathrm{HF}$, and stroke was reconstructed. The living family members (FMs) underwent cascade screening with clinical workup, including medical history, physical examination, electrocardiogram (ECG), echo, and Holter ECG monitoring. Genotype-positive patients underwent CMR. Information regarding genetic testing is presented in Supplementary material. Past medical reports, cardiac tests results, and device interrogation printouts of deceased FMs were also collected.

\section{Statistical analysis}

All calculations were done using Statistica 13PL (StatSoft, Tulsa, OK, USA). Continuous data were presented as median (range). The normality of continuous data was tested with the Shapiro-Wilk test.

\section{RESULTS AND DISCUSSION}

The pedigree tree and cardiac screening results are presented in Figure 1 and Supple- 

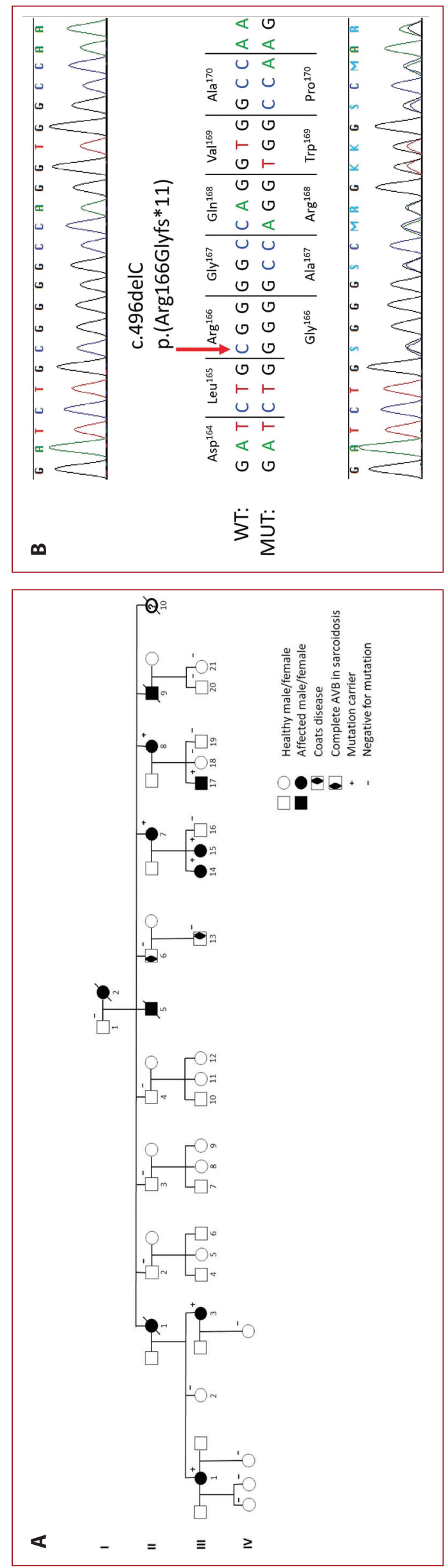

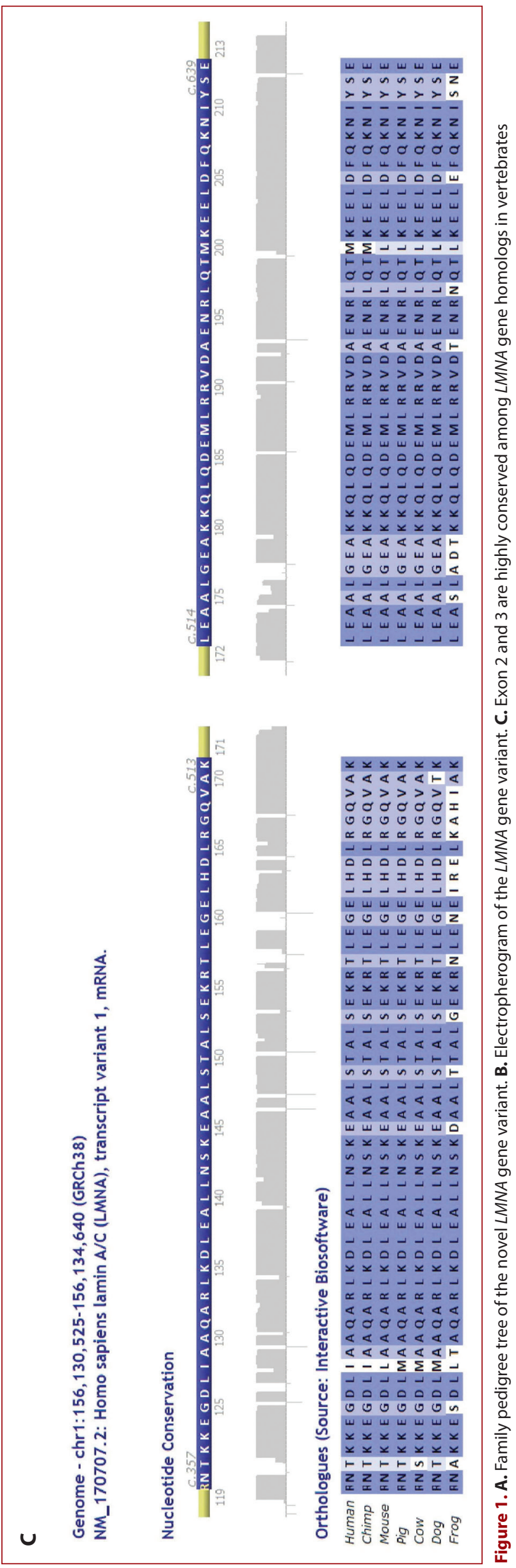


mentary material. The genotype-negative FMs were clinically asymptomatic except for patients: I-1, aged 79 presenting with atrial fibrillation (AF); II-6, implanted with a pacemaker at the age of 26 due to complete AVB and sarcoidosis; and III-4, diagnosed with Coats disease and paralysis of the perineal nerve. In 7 affected FMs, abnormal ECG findings were observed: sinus bradycardia (2), first-degree AVB (2), complete AVB (1), chronic AF (2). Supraventricular tachycardia (SVT) occurred in 7 subjects and was the first sign in teenagers. Ventricular arrhythmias, premature ventricular contractions, and nonsustained and/or polymorphic VTA were present in 8,7 , and 5 individuals, respectively. The number of premature ventricular contractions on Holter recordings was usually low (median, 5; range, 4-29 000). The youngest 16-year-old genotype-positive patient (III-17) had normal cardiac function and runs of SVT 110-160 bpm.

There were four sudden deaths; the death of a 3-monthold girl (II-10) was excluded due to incomplete data. The median age of death was 39 (range, 25-54) years. In a 54-year-old woman with AF and stroke (I-2), VTA occurred in-hospital after cholecystectomy. On autopsy, DCM was recognized. Her daughter, II-1, aged 42, died suddenly after having exertional dyspnea and leg edema for several months. Both women were considered obligate carriers. The patient II-5 was diagnosed with AF and bradycardia at the age of 34 and implanted with a VVI pacemaker followed by a cardiac resynchronization therapy defibrillator. He died several months later after the rapid progression of HF. Brother, II-9, died suddenly. His autopsy showed dilatation of both ventricles, and microscopic evaluation revealed marked scarring, peri-vascular fibrosis, and lipid metaplasia, and generalized hypertrophy of cardiomyocytes with their focal fragmentation. Since his children were genotype-negative, the local hospital was contacted to obtain preserved tissue samples. The molecular autopsy confirmed the presence of the LMNA variant in the patient's heart, proving co-segregation of the variant with cardiac phenotype.

Echocardiography revealed HNDC in 4 affected FMs. In young genotype-positive FMs, left ventricular ejection fraction (LVEF) was preserved till the age of 30 (median LVEF 59\%; range, 50\%-60\%). Patient II-5 experienced deterioration of LVEF from $42 \%$ to $21 \%$ in 5 months and died due to intractable HF.

CMR imaging was performed in 6 of 7 LMNA variant carriers; median age, 33; range, 19-44 years. The LV function and size were preserved. Myocardial fibrosis assessed by late gadolinium enhancement (LGE) was present in 3 (50\%) individuals; in 2, non-sustained VTA was also present. LGE was located midmyocardially in the basal interventricular septum and subepicardially in the LV basal postero-inferior wall. Local thinning of the LV wall was observed in 4 (67\%) patients, thus making an assessment of LGE difficult. The pattern of hypertrabeculation was found in three patients (in 2 accompanied by thin LV walls). The right ventricle was normal, except for the patient II-7 with LVEF $45 \%$.
Based on current criteria, two patients qualified for primary prevention implantable cardioverter defibrillator (ICD) [6]. The modified LMNA-VTA risk score also included patient III-3 with AVB type 1 in the high-risk group [5]. All eligible patients refused ICD implantation. The distribution of SCD risk factors is presented in Table S1.

Apart from a rise in serum creatine kinase, no overlap with other phenotypic expressions of laminopathies was observed.

\section{Genetic analysis}

Mutational analysis of the index patient II-7 revealed a heterozygous frameshift mutation in the $L M N A$ gene, denoted as LRG_254t2 (LMNA):c.496delC p.(Arg166Glyfs*11) (Figure 1). The variant was not present in the phenotype-negative brothers, the father, or brother II- 6 with complete AVB attributed to sarcoidosis. Since cardiolaminopathy may manifest as AVB, genetic testing was performed twice using two independent samples from the latter patient.

The variant was not previously described. ClinVar, NCBI dbSNP, HGMD-Public, LOVD databases do not contain an entry for this variant; its population frequency is unknown, and the alteration does not have a gnomAD exomes and genomes entry despite its locus being covered in gnomAD. The variant p.(Arg166Glyfs*11) is located in the exon 2 of the $L M N A$ gene corresponding to the coil $1 \mathrm{~B}$, an alpha-helical segment of the highly conserved central rod domain implicated in lamin A/C dimerization [8]. By disrupting the coiled-coil fold of lamin A/C, mutations affecting the structure of this domain may result in reduced nuclear rigidity and increased susceptibility to mechanical stress-induced damage [8]. As other non-missense variants, truncating mutations could induce nonsense-mediated mRNA decay resulting in haploinsufficiency. In a mouse model, the loss of half of the lamin A/C alleles recapitulates the DCM phenotype with conduction abnormalities, arrhythmias, and HF [9].

\section{Molecular autopsy}

Current guidelines advocate for collecting tissue samples for subsequent genetic testing of all unexplained SCD victims [6]. Furthermore, current molecular methods allow for the recovery of high-quality DNA from formalin-fixed, paraffin-embedded tissue. Usually, the post-mortem diagnosis allows for extending genetic screening to relatives. In this study, the molecular autopsy helped in establishing co-segregation of the LMNA variant with phenotype.

In recent years many identified DNA variants were deemed pathogenic based on case reports [7]. This resulted in harm, such as disease-labeling, insurance problems, and even implantation of unnecessary defibrillators [7]. As the final interpretation of genetic workup should be based on integrated genetic and clinical data, we performed clinical and genetic screening, searched for medical data of the deceased, and performed the molecular autopsy of the last SCD victim. The co-segregation analysis allowed for 
establishing the genotype-phenotype relation in relatives, presenting ECG abnormalities and SVT in young adults, HNDC in middle-aged patients, and DCM in later stages of the disease. In line with previous reports, we observed complete penetrance of the laminopathy [3]. According to our expertise, the following ACMG/AMP criteria were fulfilled: PVS1, PM2, PP1 to classify the variant as pathogenic [10].

\section{SCD and stroke risk}

Although the presence of truncating mutations increases the risk of malignantVTA in risk stratification schemes [3-5], the genotype-positive ICD-eligible FMs refused an ICD. Several tools were proposed to increase the acceptance of ICD therapy e.g., the Implantable Cardioverter-Defibrillator Concerns questionnaire, addressing causes of patients' anxiety [11]. In case of the lack of acceptance for a conventional ICD, the wearable cardioverter-defibrillator can be prescribed [12]. However, its high cost and difficulties in reimbursement in Poland, as well as potentially low rates of patient adherence, which is a key driver of the efficacy of wearable cardioverter-defibrillator in long-term treatment, limit its use.

Patients with cardiolaminopathy have a high risk of cardioembolic stroke, irrespective of $\mathrm{CHA}_{2} \mathrm{DS}_{2}$-VASc score, dependent on the occurrence of AF, low LVEF, and/or the presence of non-compacted myocardium. In such cases, anticoagulation could be initiated based on the indications applied in the LV non-compaction cardiomyopathy [13].

\section{CONCLUSIONS}

The novel variant c.496delC $p$.(Arg166Glyfs*11) in the LMNA gene is associated with the highly malignant phenotype of SCD and DCM/ HNDC. The molecular autopsy allowed for assigning pathogenicity to the variant in the presence of complete AVB as a confounder. There is an ongoing need to preserve tissue samples of SCD victims to allow for molecular autopsy and reimbursement of the method.

\section{Supplementary material}

Supplementary material is available at https://journals. viamedica.pl/kardiologia_polska.

\section{Article information}

Conflict of interest: None declared.

Open access: This article is available in open access under Creative Common Attribution-Non-Commercial-No Derivatives 4.0 International (CC BY-NC-ND 4.0) license, allowing to download articles and share them with others as long as they credit the authors and the publisher, but without permission to change them in any way or use them commercially. For commercial use, please contact the journal office at kardiologiapolska@ptkardio.pl.
How to cite: Zienciuk-Krajka A, Chmara M, Lica-Gorzyńska M, et al. The novel pathogenic variant in the LMNA gene in a four-generation family with sudden deaths and cardiomyopathy: Utility of molecular autopsy. Kardiol Pol. 2021; 79(12): 1368-1371, doi: 10.33963/KP.a2021.0148.

\section{REFERENCES}

1. Fatkin D, MacRae C, Sasaki T, et al. Missense mutations in the rod domain of the lamin $\mathrm{A} / \mathrm{C}$ gene as causes of dilated cardiomyopathy and conduction-system disease. N Engl J Med. 1999; 341(23): 1715-1724, doi: 10.1056/NEJM199912023412302, indexed in Pubmed: 10580070.

2. Pinto YM, Elliott PM, Arbustini E, et al. Proposal for a revised definition of dilated cardiomyopathy, hypokinetic non-dilated cardiomyopathy, and its implications for clinical practice: a position statement of the ESC working group on myocardial and pericardial diseases. Eur Heart J. 2016; 37(23): 1850-1858, doi: 10.1093/eurheartj/ehv727, indexed in Pubmed: 26792875.

3. van Rijsingen IAW, Arbustini E, Elliott PM, et al. Risk factors for malignant ventricular arrhythmias in lamin a/c mutation carriers a European cohort study. J Am Coll Cardiol. 2012; 59(5): 493-500, doi: 10.1016/j. jacc.2011.08.078, indexed in Pubmed: 22281253.

4. Nishiuchi S, Makiyama T, Aiba T, et al. Gene-based risk stratification for cardiac disorders in mutation carriers. Circ Cardiovasc Genet. 2017; 10(6): e001603, doi: 10.1161/CIRCGENETICS.116.001603, indexed in Pubmed: 29237675.

5. Wahbi K, Ben Yaou R, Gandjbakhch E, et al. Development and validation of a new risk prediction score for life-threatening ventricular tachyarrhythmias in laminopathies. Circulation. 2019; 140(4): 293-302, doi: 10.1161/CIRCULATIONAHA.118.039410, indexed in Pubmed: 31155932.

6. Priori S, Blomström-Lundqvist C, Mazzanti A, et al. 2015 ESC Guidelines for the management of patients with ventricular arrhythmias and the prevention of sudden cardiac death. Eur Heart J. 2015; 36(41): 2793-2867, doi: 10.1093/eurheartj/ehv316, indexed in Pubmed: 26320108.

7. Ackerman MJ. Genetic purgatory and the cardiac channelopathies: exposing the variants of uncertain/unknown significance issue. Heart Rhythm. 2015; 12(11): 2325-2331, doi: 10.1016/j.hrthm.2015.07.002, indexed in Pubmed: 26144349.

8. Carmosino M, Torretta S, Procino $G$, et al. Role of nuclear Lamin A/C in cardiomyocyte functions. Biol Cell. 2014; 106(10): 346-358, doi: 10.1111/boc.201400033, indexed in Pubmed: 25055884.

9. Wolf CM, Wang L, Alcalai R, et al. Lamin A/C haploinsufficiency causes dilated cardiomyopathy and apoptosis-triggered cardiac conduction system disease. J Mol Cell Cardiol. 2008; 44(2): 293-303, doi: 10.1016/j. yjmcc.2007.11.008, indexed in Pubmed: 18182166.

10. Richards S, Aziz N, Bale S, et al. ACMG Laboratory Quality Assurance Committee. Standards and guidelines for the interpretation of sequence variants: a joint consensus recommendation of the American College of Medical Genetics and Genomics and the Association for Molecular Pathology. Genet Med. 2015; 17(5): 405-424, doi: 10.1038/gim.2015.30, indexed in Pubmed: 25741868.

11. Młynarska A, Młynarski R, Uchmanowicz I, et al. Crosscultural adaptation and reliability testing of the Implantable Cardioverter-Defibrillator Concerns questionnaire to optimize the care of Polish patients with implantable cardioverter-defibrillators. Kardiol Pol. 2020; 78(9): 906-912, doi: 10.33963/KP.15520, indexed in Pubmed: 32692027.

12. Sterliński M, Oręziak A, Przybylski A, et al. Experts of the Heart Rhythm Section of the Polish Cardiac Society: opinion on the use of wearable cardioverter-defibrillators in Poland. Kardiol Pol. 2019; 77(2): 238-243, doi: 10.5603/KP.2019.0031, indexed in Pubmed: 30816992.

13. Zienciuk-Krajka A, Daniłowicz-Szymanowicz L, Dorniak K, et al. Left ventricular noncompaction cardiomyopathy: diagnostic and therapeutic dilemmas. Kardiol Pol. 2020; 78(10): 1053-1054, doi: 10.33963/KP.15503, indexed in Pubmed: 32640777. 